\title{
LASER TONSIL CRYPTOLYSIS: PERSPECTIVES IN AIRBORNE PERSONNEL
}

\author{
Rafał CHMIELEWSKI \\ Military Institute of Aviation Medicine, Department of Otolaryngology, Warsaw, Poland
}

Source of support: Own sources

Author's address: R. Chmielewski, Military Institute of Aviation Medicine, Department of Otolaryngology, Krasińskiego 54/56 Street, 01-755 Warsaw, Poland, e- mail: rchmiele@wiml.waw.pl

Background: Laser tonsil cryptolysis is a relatively new, minimally-invasive method of chronic caseous tonsillitis and oral malodor treatment. Recently, several reports were published presenting this technique and its clinical outcomes. This review article presents the techniques and the clinical results of the treatments reported in published articles available in the PubMed database.

Keywords: laser cryptolysis, chronic caseous tonsillitis, tonsil stones, tonsilloliths, bad breath, halitosis

\section{INTRODUCTION}

Odors are one of the most important signals we collect from our environment, as it may provide warning of dangers, information on surrounding objects, revoke memories of the past as well as encourage or discourage us from interacting with other people. Aircraft pilots and, even more than that, flight attendants, are occupations that involve direct contact with clients. It is quite easy to imagine the negative impact of oral malodor in the deck crew on the relationships with the passengers and thus on the overall opinion of flight quality. The goal of this article is to present the results of the treatment of oral malodor reports due to chronic palatine tonsillitis by means of laser cryptolysis as well as the applicability of this method in the treatment of airborne flight personnel.

Tonsils are covered with multilayer epithelium forming tonsillar crypts within the parenchyma. Each palatine tonsil has 10 to 20 crypts. These spaces become anaerobic chambers that promote the growth of the biofilm of particular bacterial strains. As the biofilm grows and matures, it dilates

Figures: 3 - Tables: 1 - References: 10 - Full-text PDF: http://www.pjamp.com • Copyright (C 2013 Polish Aviation Medicine Society, ul. Krasińskiego 54/56, 01-755 Warsaw, license WIML • Indexation: Index Copernicus, Polish Ministry of Science and Higher Education 
the crypt, leading to formation of tonsil stone and inflammation in its vicinity.

Tonsil stones have several equivalent names in both English and Polish nomenclature. In English, they may be called tonsilloliths or cryptic tonsils. A common Polish name is "detryt". One of first modern articles providing an attempt to analyze tonsil stones defined them as purulent content with dead bacteria and inflammatory cells within the tonsillar crypts following an infection [10]. The largest tonsil stone described in the literature weighed 44 grams [9]. A new look on the structure and physiology of tonsil stones was provided by Stoodley who, having meticulously studied their contents, compared them to biofilm deposits on the teeth. The biofilm develops in tissue hollows and fissures and consists of the matrix and bacteria in slow-metabolism forms, capable of leaving hibernation in favorable external conditions. These consist mostly of anaerobic Gram-negative bacterial strains.

Tonsillar complaints and symptoms reported in the literature include a feeling of a presence of a foreign body, metallic taste in mouth, pharyngeal constriction or compression, cough, choking, and - most of all - bad breath, also known as oral malodor or halitosis. By itself, oral malodor may be caused by numerous causes. Oral causes are estimated to account for about $90 \%$ of cases. In $8 \%$, the etiology of the disorder is in the nose and throat or associated with the respiratory system, while the remaining $2 \%$ include other causes, such as gastrointestinal disorders, renal or hepatic insufficiency of metabolic disorders, including dental caries, gingivitis, disorders within paranasal sinuses, lung or esophagus (mainly the Zenker's diverticulum) [7].

In recent years, the range of diagnostic methods was widened by an objective method to assess oral malodor: halitometry. The method consists in the measurement of volatile sulfur compounds (VSC) in the exhaled air. According to the manufacturer of the device, VSC values that exceed the limit of normal are those of > 150 ppb. Hitherto, researchers used only subjective assessments of patient's oral odor, which negatively affected the scientific value of the assessment. A subjective assessment technique, known as Finkelstein's tonsil smelling test, was described by Finkelstein and widely adopted by the researchers [3]. The method consists in the assessment of the odor of secretion from the palatine tonsil. The tester presses the tonsil with a gloved finger for further organoleptic assessment by the tester themselves, the patient, and individuals close to the patient. The goal of this assessment is to establish whether the smell is consistent with the usual smell of patient's mouth. The intensity of halitosis is expressed in a three-point scale: severe (when oral malodor can be smelled during conversation with the patient), moderate (when strong, unpleasant odor is smelled on the glove at a distance of $10 \mathrm{~cm}$ or while smelling the air exhaled by the patient) or mild, when no odor is smelled at patient's mouth and the odor of the glove is detected only upon placing it immediately close to the nostrils.

Recently, a specific Halitosis Associated Lifequality Test questionnaire (HALT) has been developed and validated in psychological and clinical terms by Kizhner et al. [4]. The test has been developed from scratch based on patient interviews and the review of the literature. The questionnaire contains 20 halitosis-specific questions (Tab. 1.). The authors compared the results of subjective organoleptic assessments with HALT results obtained upon subsequent visits and then following 3 months of conservative treatment laser cryptolysis depending on the study group. The results of the HALT test were assessed to be significantly correlated with the results of the organoleptic test and that HALT allowed for correct assessment of the treatment outcomes in both groups, thus constituting a reliable tool for the assessment of the quality of life in patients complaining of bad breath.

Tab. 1. HALT questionnaire - adapted from (Kizhner et al., 2011).

\begin{tabular}{cl}
\hline Q1. & Mainly mouth breathing \\
\hline Q2. & Frequent tonsillar infections \\
\hline Q3. & Frequent sinus infections \\
\hline Q4. & Worrying about or self-conscious about your mouth breath \\
\hline Q6. & Miserable or tense due to halitosis \\
\hline Q7. & Difficulty chewing or limiting certain food due to halitosis \\
\hline Q8. & Problemse of taste \\
\hline Q9. & Appearance affected due to halitosis \\
\hline Q10. & Depressed due to mouth breath \\
\hline Q11. & Problems concentrating due to halitosis \\
\hline Q12. & Embarrassed due to halitosis \\
\hline Q13. & Spending time related to halitosis? \\
\hline Q14. & Talking from afar due to halitosis \\
\hline Q15. & Avoid going out due to halitosis \\
\hline Q16. & Communication problems due to halitosis \\
\hline Q17. & Mentioned about halitosis \\
\hline Q18. & Suffer financial loss due to halitosis \\
\hline Q19. & Suffer social/personal loss due to halitosis \\
\hline Q20. & Reduced life satisfaction due to halitosis \\
\hline
\end{tabular}


R. Chmielewski - Laser tonsil...

\section{OPERATIVE TECHNIQUE}

The premise of laser cryptolysis is minimallyinvasive treatment of cryptic tonsils and halitosis while preserving its immune function. Another important factor is that the procedure is performed in an outpatient setting under local anesthesia. This shortens the duration of the procedure and significantly reduces associated costs. Yet another benefit is that the treatment can be repeated if complete improvement has not been achieved.
Local anesthesia is usually sufficient to eliminate the gag reflex, either infiltrative or topical. A CO2 laser beam (some authors have also used diode lasers in cases with single crypt retention) is applied onto the surface of the tissue surrounding the tonsillar crypt in combination with a $2 \mathrm{~mm}$ rotation scanner to vaporize the sequential layers. Shrinking tissue in the crypt mouth region causes an outward movement of the crypt edges, leading to marsupialization, i.e. wide opening of the crypt.

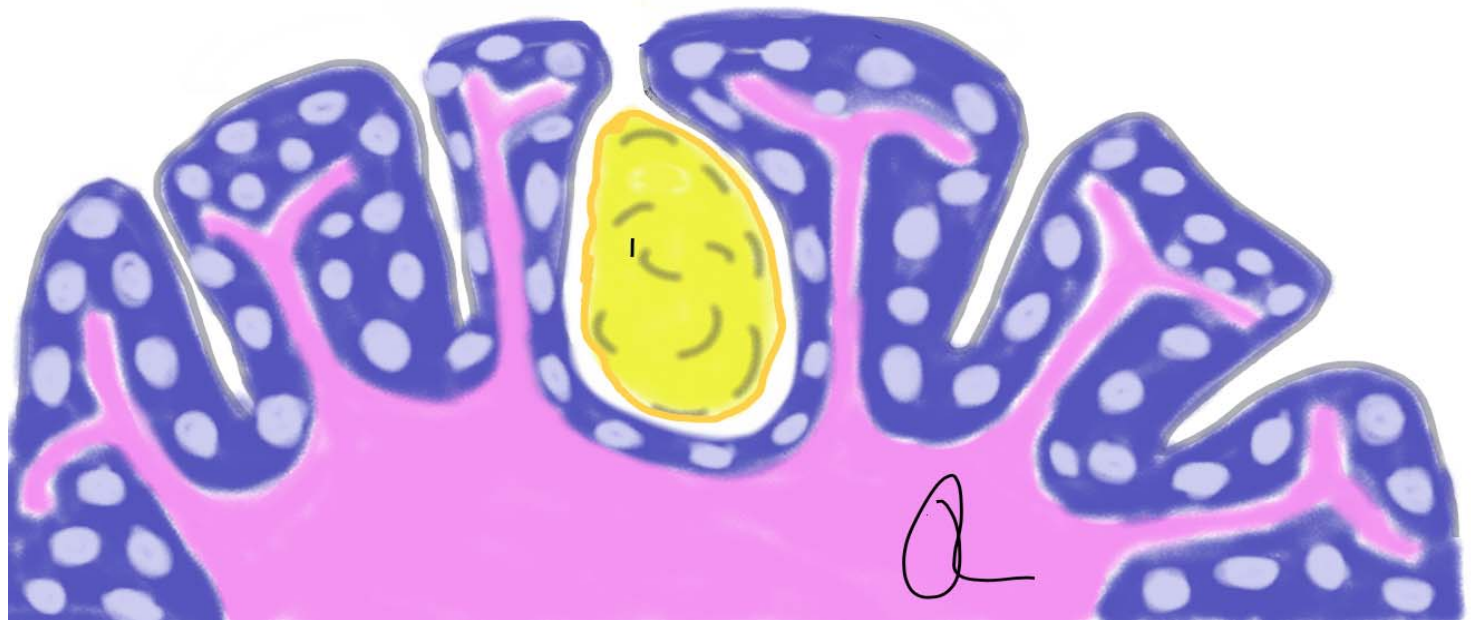

Fig. 1. A schematic illustration of a tonsillar stone in a dilated palatine tonsillar crypt. Author's diagram based on Y. P. Krespi \& Kizhner, 2013.

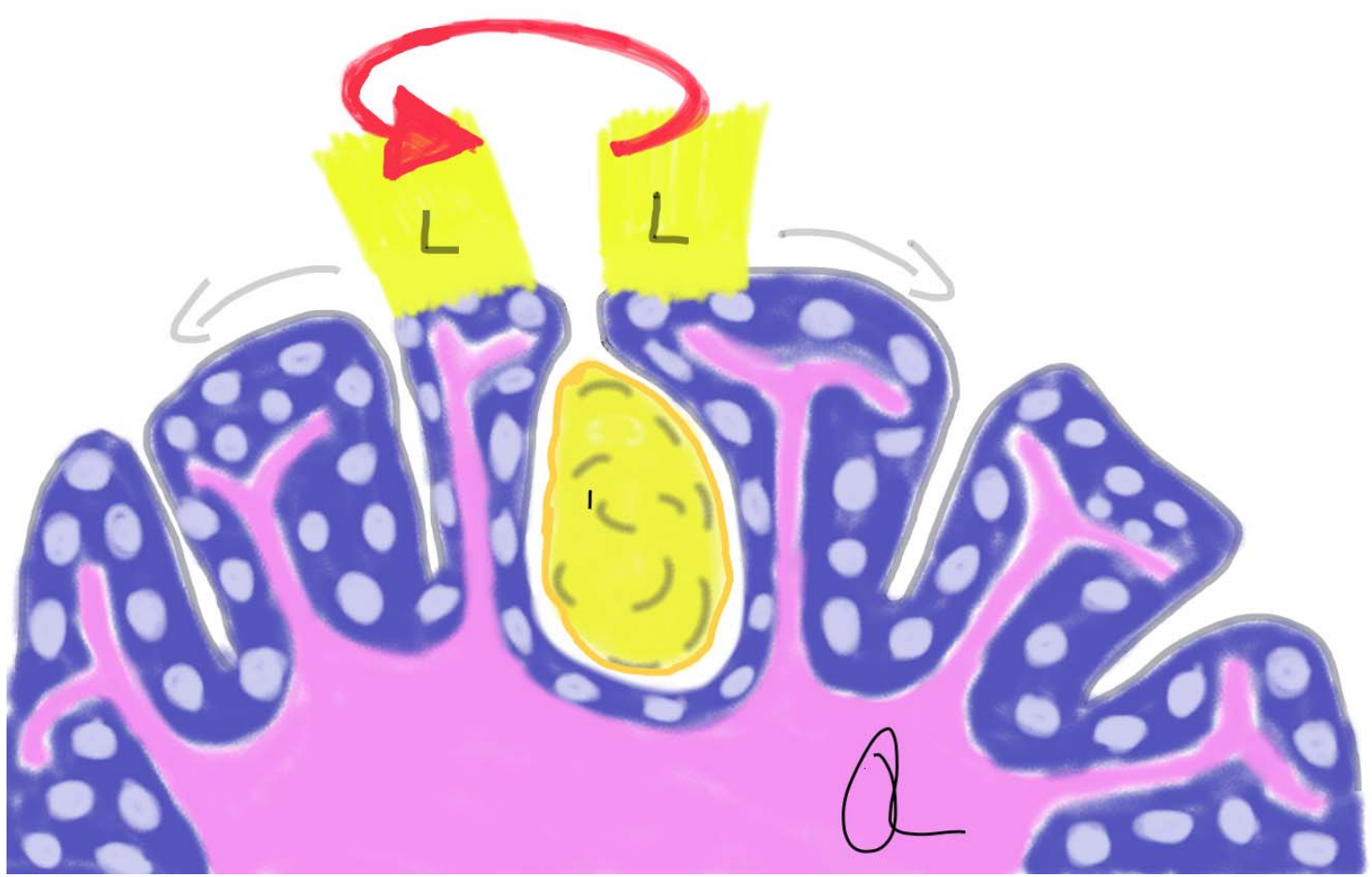

Fig. 2. A schematic illustration of laser (L) vaporization of the palatine tonsil crypt mouth region. Combination of the laser beam with the scanner facilitates superficial vaporization of following epithelial layers until complete opening of the crypt. The immediately adjacent tonsil tissue shrinks (arrows pointing sideways), additionally opening the mouth of the crypt. Author's diagram based on Y. P. Krespi \& Kizhner, 2013. 


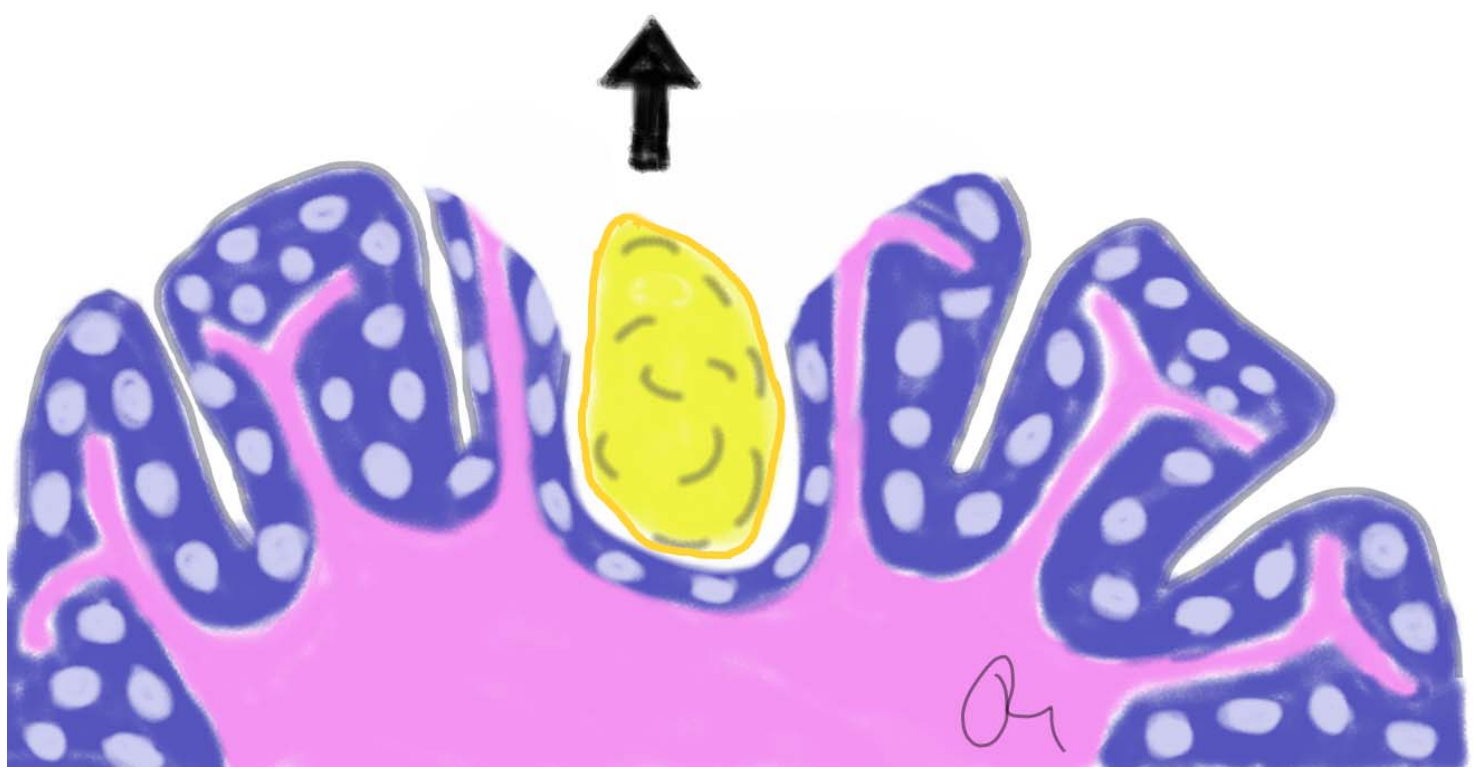

Fig. 3. Removal of a tonsil stone from a widely open crypt following laser cryptolysis. Author's diagram based on Y. P. Krespi \& Kizhner, 2013.

As the laser beam reaches the bottom of the crypt, it facilitates its full emptying and shallowing. Coagulation of the crypt bottom is necessary to make the procedure effective and to prevent formation of new tonsillar stones. No cases of intraoperative bleeding have been reported. Following the procedure, patients are administered antibiotics and local analgesics. Cryptolysis procedure may be repeated several times since it is not efficient enough initially.

\section{LITERATURE REVIEW}

The initial reports on cryptic stones date back to the 1970s [10]. Since that time, they were an object of attention in descriptional and therapeutic aspects, and the methods of their treatment evolved together with the technological advances in medicine. The first report describing $\mathrm{CO}_{2}$ laser cryptolysis was published by Krespi 20 years ago [6]. Since that time, the developments pertained mostly to three aspects: establishment of objective, repeatable diagnostic methods, selection of suitable patient groups in whom best outcomes could be achieved after the treatment and, finally, the assessment of the efficacy and its effect on the patients' quality of life.

Finkelstein et al. evaluated palatine tonsils as the source of oral malodor and the efficacy of $\mathrm{CO}_{2}$ laser cryptolysis in the treatment of oral malodor caused by chronic caseous tonsillitis [3]. A total of 53 patients with halitosis of tonsillar origin, subjected to the cryptolysis procedure were qualified for the study. The assessment of oral malodor was subjective and performed according to the Finkelstein's tonsil smelling test as developed by the author. The test consisted in organoleptic assessment of the secretion from a compressed tonsil. Post-operative assessment was based on the clinical assessment and the interviews with patients and their families conducted 4-6 weeks after the treatment. Complete resolution of halitosis required one cryptolysis session in 28 patients (52.8\%), two sessions in 18 patients (34\%) and three sessions in five patients (9.4\%). The follow-up period ranged from 3 to 36 months (mean of 20.8). No side effects or treatmentemergent adverse events were observed.

In 2004, Passos et al. performed a histological assessment of specimens collected before and after $\mathrm{CO}_{2}$ laser cryptolysis [8]. The authors assessed the germinal centers, lymphatic tissue and the degree of subepithelial and interstitial fibrosis. The technique of the procedure was analogous to that described above by Krespi and Ling [6]. The authors observed that the procedure caused no atrophy of the tonsil lymphatic tissue and superficially coagulated the epithelial tissue, promoting the opening of tonsillar crypts.

The study by Dal Rio et al. described the results of the treatment of 38 patients complaining of bad breath [2]. Patients were subjected to physical examination, halitometry and 4 sessions of laser cryptolysis. The protocol and instrument settings were as in the remaining studies. Halito- 
metry measurements were performed before each laser cryptolysis session. The procedures were well-tolerated and all patients experienced the improvement of symptoms. Prior to the study, 8 patients (21\%) had abnormal halitometry results (>150 ppb); all halitometry values returned to normal following the treatment. The average reduction in the volatile sulfur compounds levels in the study was $30.1 \%$ and was accompanied by significant reduction in tonsillar stone deposits within the crypts. The results positively summarized the safety and the efficacy of laser cryptolysis.

A similar study with patients being classified into groups depending on their halitometry results was conducted by Dal Rio et al. [1]. The study included a total of 49 patients, 17 males (35\%) and 32 females (65\%), who were recruited to group A - normal halitometry results (41 patients, $83.7 \%$ ) and group B - halitometry result $>150 \mathrm{ppb}(8 \mathrm{pa}-$ tients, $16.3 \%$ ) following baseline evaluation. Tonsillar stones were present in $75 \%$ of patients with abnormal halitometry results and in as little as $6 \%$ of patients with normal halitometry results. Thus, it was found that the presence of tonsilloliths increased tenfold the risk of bad breath.

The largest study material to date was published this year by a New York research group in the American Journal of Otolaryngology - Head and Neck Medicine and Surgery [5]. The authors described the results from 500 consecutive patients treated from 2003 to 2011 with laser cryptolysis for chronic tonsillitis with or without cryptic stones. Qualified for the procedure were only cooperating patients with tonsil sizes not exceeding +2 , with mild or moderate gag reflexes and with visible oropharynx. Subjects with tonsils crossing the palatopharyngeal arch, over-reactive gag reflexes and non-cooperating were excluded from the study. The procedures were performed under local topical and infiltrative anesthesia. Starting from 2010, a group of 50 patients additionally completed the HALT questionnaire before and after the treatment. Location of tonsilloliths within the tonsils was also assessed. As compared to the operative technique described by Krespi and Ling [6], the authors decided to perform additional ablation of the upper segment of the palatoglossal arch to expose crypts located at the upper pole of the tonsil.

Patients' age ranged from 11 to 73 years, with women prevailing in the study group (62\%). The procedure duration was shorter than 30 minutes. In most procedures, authors made use of an $18 \mathrm{~W}$ $\mathrm{CO}_{2}$ laser with a $2 \mathrm{~mm}$ scanner in continuous operation mode. If the procedure was to be performed on a single crypt, a 10W $980 \mathrm{~nm}$ diode laser was used, also in continuous operation mode. The first follow up examination after the procedure was scheduled between days 4 and 9. Post-operative bleeding, immediately managed in the outpatient setting under local anesthesia was observed in one case. Five patients reported minor postoperative bleeding that resolved spontaneously after drinking some cold water. None of the patients required emergency hospitalization. A total of 80 patients required the procedure to be repeated due to persistent symptoms, with the average 1.16 procedures per patient. In 18 patients (3.6\%), full recovery required tonsillectomy under general anesthesia. The overall patient satisfaction was very high, with 0-2 days on sick leave from work. In 50 patients undergoing the procedure since 2010, in whom cryptic stone location and HALT questionnaire results were also assessed, stones were invisible in $40 \%$ of cases (hidden behind the palatoglossal arch) and the improvement in questionnaire results was up to $46 \%(P<0.05)$.

In the discussion, the authors highlighted the importance of full tonsil exposure by means of retracting the palatoglossal arch which might result in an additional $250 \%$ increase in exposure of the tonsil surface and a much more complete assessment of crypts, and thus the increased efficacy of the procedure. In the conclusions, authors suggest that laser cryptolysis may frequently be an alternative to tonsillectomy providing proper selection of patients.

\section{SUMMARY}

The results of $\mathrm{CO}_{2}$ laser cryptolysis treatment available in papers published to date allow for a favorable assessment of the efficacy and safety of the method. Of note is the fact that provided proper selection and qualification for laser cryptolysis, the percentage of patients requiring tonsillectomy for full recovery is very small. Another benefit of laser cryptolysis treatment is conservation of the immune function of the palatine tonsils, which may be particularly important in young patients. A disadvantage of the method is in its inability to cure chronic inflammation located deep within tonsillar parenchyma, which should be taken into consideration by physicians qualifying patients for laser cryptolysis or tonsillectomy.

Laser cryptolysis appears to be a favorable therapeutic option for airborne personnel, particularly due to its minimum invasiveness and short procedure and recovery times, which is important in light of the strained work schedules of aircraft pilots and flight attendants. 
Review Article

\section{AUTHORS' DECLARATION:}

Study Design: Rafał Chmielewski; Data Collection: Rafał Chmielewski; Manuscript Preparation: Rafał Chmielewski; Funds Collection: Rafał Chmielewski. The Author declares that there is no conflict of interest.

\section{REFERENCES}

1. Dal Rio, A. C., Franchi-Teixeira, A. R., Nicola, E. M. (2008) Relationship between the presence of tonsilloliths and halitosis in patients with chronic caseous tonsillitis. Br Dent J. 204 (2). E4. doi: 10.1038/bdj.2007.1106

2. Dal Rio, A. C., Passos, C. A., Nicola, J. H., Nicola, E. M. (2006) $\mathrm{CO}_{2}$ laser cryptolysis by coagulation for the treatment of halitosis. Photomed Laser Surg. 24 (5). 630-636. doi: 10.1089/pho.2006.24.630

3. Finkelstein, Y., Talmi, Y. P., Ophir, D., Berger, G. (2004) Laser cryptolysis for the treatment of halitosis. Otolaryngol Head Neck Surg. 131 (4). 372-377. doi: 10.1016/j.otohns.2004.02.044

4. Kizhner, V., Xu, D., Krespi, Y. P. (2011) A new tool measuring oral malodor quality of life. Eur Arch Otorhinolaryngol. 268 (8). 1227-1232. doi: 10.1007/s00405-011-1518-x

5. Krespi, Y. P., Kizhner, V. (2013) Laser tonsil cryptolysis: In-office 500 cases review. Am J Otolaryngol. 34 (5). $420-424$. doi: 10.1016/j.amjoto.2013.03.006

6. Krespi, Y. P., Ling, E. H. (1994) Tonsil cryptolysis using $\mathrm{CO}_{2}$ Swiftlase. Oper Tech in Otol-Head and Neck Surg. 5 (4). $294-$ 297.

7. Lu, D. P. (1982) Halitosis: an etiologic classification, a treatment approach, and prevention. Oral Surg Oral Med Oral Pathol. 54 (5). 521-526.

8. Passos, C. A., Altemani, A., Nicola, J. H., \& Nicola, E. M. (2004) Histopathological evaluation in biopsies of palatine tonsils submitted to cryptolysis by coagulation with $\mathrm{CO} 2$ laser for treatment of chronic caseous tonsillitis. Photomed Laser Surg. 22 (3). 211-219. doi: 10.1089/1549541041438605

9. Pruet, C. W., Duplan, D. A. (1987) Tonsil concretions and tonsilloliths. Otolaryngol Clin North Am. 20 (2). $305-309$.

10. Samant, H. C., Gupta, O. P. (1975) Peritonsillolith. Oral Surg Oral Med Oral Pathol. 40 (1). 56-60.

Cite this article as: Chmielewski R: Laser tonsil cryptolysis: perspectives in airborne personnel. Pol J Aviat Med Psychol, 2013; 19(2): 25-30. 